\title{
A relação da perda auditiva com tontura e zumbido na população idosa
}

\author{
The relationship of hearing loss with dizziness \\ and tinnitus in the elderly population
}

\section{La relación entre la pérdida auditiva, mareos $y$ tinnitus en adultos mayores}

\author{
Ana Carla Oliveira Garcia* \\ Aline Cabral Oliveira* \\ Barbara Cristina da Silva Rosa** \\ Teresa Maria Momensohn-Santos**
}

\section{Resumo}

Introdução: A perda auditiva relacionada a sintomas de tontura e zumbido pode ser um fator de limitação do idoso com consequências psicossociais. Com o decorrer da idade as alterações vestibulares e outros sintomas auditivos associados aumentam. Objetivos: Verificar a relação da perda auditiva e queixas relacionadas à tontura, zumbido e distúrbios da comunicação em uma população de idosos. Método: Trata-se de um estudo retrospectivo que contou com análise de 468 registros de idosos cadastrados no período de 2011 a 2015 em Serviço de Reabilitação Auditiva. Foram coletados dados referentes à idade, sexo, queixas auto relatadas de zumbido, dificuldade de compreensão de fala e tontura, bem como os resultados da avaliação audiométrica, simetria entre as orelhas no caso de perda auditiva, grau de deficiência auditiva e lateralidade. Resultado: A amostra foi composta por 295 (63\%) prontuários de sujeitos do sexo feminino e 173 (37\%) do sexo masculino com idade média de 72,7 anos. A queixa "ouvir e não compreender" foi encontrado em $64,7 \%$ dos idosos seguido por queixa de zumbido $(45,1 \%)$ e tontura (20,3\%). Observou-se que a tontura tem relação significativa com queixar-se de não entender a fala $(\mathrm{p}<0,001 ; \mathrm{R}=0,17)$ e presença de zumbido $(\mathrm{p}<0,001 ; \mathrm{R}=0,32)$. Em $77,7 \%$ dos prontuários houve o

\footnotetext{
*Universidade Federal de Sergipe, Aracaju, SE, Brasil.

**Pontifícia Universidade Católica de São Paulo, São Paulo, SP, Brasil.

Contribuição dos autores:

ACOG: revisão de literatura, coleta dos dados nos prontuários, redação do artigo científico, aprovação da versão final do conteúdo a ser publicado.

ACO: análise estatística e aprovação da versão final do conteúdo a ser publicado.

BCSR: revisão de literatura, redação do artigo científico, aprovação da versão final do conteúdo a ser publicado.

TMMS: revisão do artigo e aprovação da versão final do conteúdo a ser publicado.
}

E-mail para correspondência: Ana Carla Oliveira Garcia - anacarlagarciausa@gmail.com Recebido: $24 / 06 / 2016$

Aprovado: $12 / 03 / 2017$ 


\section{Abstract}

Introduction: Hearing loss related to symptoms of dizziness and tinnitus may be a limiting factor reported by elderly population with psychosocial consequences. Objectives: To verify the relationship between hearing loss and complaints related to dizziness, tinnitus and communication disorders in the elderly population. Method: This is a retrospective study that included the analysis of 468 records of elderly people enrolled in the period from 2011 to 2015 in Rehabilitation Service. Data regarding age, gender, self-reported complaints of tinnitus, difficulty in understanding speech and dizziness, as well as the results of audiometric evaluation (presence or absence of hearing loss), symmetry and degree of hearing loss and laterality were collected. Results: The sample consisted of $295(63 \%)$ records of female subjects and $173(37 \%)$ males with a mean age of 72.7 years. The complaint "listen and not understand" was found in $64.7 \%$ of the elderly followed by complaint of tinnitus $(45.1 \%)$ and dizziness $(20,3 \%)$. It has been observed that dizziness has a significant relationship with complaining about not understanding speech ( $\mathrm{p} .0,001 ; \mathrm{R}=0,17$ ) and between tinnitus and dizziness. $(\mathrm{p}, 0,001 ; \mathrm{R}=0,32)$. It can be observed that $77.7 \%$ of the subjects presented losses classified as moderate to moderately severe, symmetrical $(80.6 \%)$ and bilateral (96.8\%). Conclusion: Based on these findings, local managers will be able to prepare adequate management to improve the quality of service and life of this elderly population.

Keywords: Hearing Loss; Dizziness; Tinnitus.

\section{Resumen}

Introducción: La pérdida auditiva relacionada con síntomas de vértigo y tinnitus puede ser un factor limitante en adultos mayores con consecuencias psicosociales. Con el aumento de la edad las alteraciones vestibulares y otros síntomas auditivos asociados aumentan. Objetivos: Verificar la relación entre pérdida auditiva y quejas relacionadas a vértigo, tinnitus y disturbios de la comunicación en una población de adultos mayores. Método: Se realizó un estudio retrospectivo que incluyó el análisis de 468 registros de adultos mayores, matriculados en el período de 2011 a 2015 en los Servicios de Rehabilitación Auditiva. Se recogieron datos en cuanto a edad, sexo, quejas auto relatadas de tinnitus, dificultades de comprensión del habla y vértigo, así como los resultados de la evaluación audiométrica, simetría entre los oídos en los casos de perdida auditiva, grado de la pérdida auditiva y lateralidad. Resultados: La muestra estuvo constituida por $295(63 \%)$ registros de sujetos del sexo femenino y $173(37 \%)$ del sexo masculino, con un promedio de edad de 72,7 años. La queja "oir y no entender" se encontró en el $64,7 \%$ de los adultos mayores, seguido por la queja de tinnitus $(45,1 \%)$ y vértigo $(20,3 \%)$. Se observó que el vértigo tiene una relación significativa con la queja de no entender el habla ( $\mathrm{p}<0,001, \mathrm{R}=17$ y el tinnitus $(\mathrm{p}<0,001, \mathrm{R}=0,32)$. En el $77,7 \%$ de los registros se encontró anotaciones a respeto de pérdidas clasificadas de moderadas a moderadamente severa, de tipo simétrico $(80,6 \%)$ y bilateral $(96,8 \%)$. Conclusión: Con base en esto allazgos, los administradores locales podrán diseñar una planificación adecuada con el fin de mejorar la calidad del servicio y la vida de esta población de adultos mayores.

Palabras claves: Pérdida Auditiva; Vértigo; Tinnitus 


\section{Introdução}

A Política Nacional de Saúde da Pessoa Idosa instituída pelo Ministério da Saúde assumiu com as esferas municipais e estaduais de saúde, o compromisso em investir na qualidade de atenção prestada pelo Sistema Único de Saúde (SUS) às pessoas com mais de 60 anos, uma vez que projeções apontam para o fato de que, em 2025, o Brasil será a sexta maior população idosa do mundo ${ }^{1}$.

Segundo o National Institute on Deafness and Other Communication Disorders (NIDCD), 30 a $35 \%$ dos adultos com idades entre 65 e 70 anos apresentam algum grau de perda auditiva e essa porcentagem sobe para 40 a $50 \%$ nos indivíduos com mais de 75 anos. Infelizmente, um grande número de pessoas idosas não recebe o tratamento adequado para essa deficiência o que pode afetar negativamente as atividades de vida diária. A associação entre a severidade da deficiência auditiva relacionada à idade e o comprometimento das atividades cotidianas foi pesquisada e constatada em 1952 participantes com mais de 60 anos de idade 2 .

Em especial no Brasil, os idosos podem contar com a Política Nacional de Atenção à Saúde Auditiva (PNASA) que propicia a avaliação e posterior concessão de aparelho de amplificação sonora individual (AASI).

O uso do Aparelho de Amplificação Sonora (AASI) pode contribuir de forma positiva para a "recuperação" dessa capacidade, pois ao melhorar a qualidade e a intensidade do sinal periférico, favorece o processamento dessa informação até que esse alcance o sistema cognitivo ${ }^{3}$.

Pesquisadores estudaram se o uso de AASI está associado com melhor desempenho cognitivo, e com relação ao isolamento social e/ou depressão, em amostra composta por 164.770 adultos do Reino Unido, entre 40 e 69 anos, que realizaram avaliação audiológica completa. Esta pesquisa constatou que o uso do AASI esteve associado a uma melhora da cognição, independentemente do isolamento social e depressão. Essa constatação foi consistente com a hipótese de que o AASI pode melhorar o desempenho cognitivo ${ }^{4}$.

Uma queixa que a população idosa apresenta é a dificuldade para ouvir e compreender a conversação. Em pesquisa realizada com essa população foi registrado que ouvintes mais velhos precisam de relação sinal-ruído de mais de $3 \mathrm{~dB}(\mathrm{~S} / \mathrm{R}+3)$ do que os adultos mais jovens devido ao envelheci- mento auditivo e processamento temporal auditivo possivelmente mais lento 5 .

Em um estudo realizado em São Paulo, por meio do levantamento de prontuários de 191 idosos encaminhados para o uso de AASI, no período compreendido entre outubro de 2012 e janeiro de 2013, com idosos com idade superior a 60 anos, que tinha como objetivo identificar o perfil populacional de idosos encaminhados à seleção de próteses auditiva demonstrou que em relação à percepção auditiva, 96\% relataram alguma dificuldade na audição, sendo que 94 (51\%) relataram como dificuldade regular e 81 (44\%), como ruim Desta amostra, $55 \%$ apresentava perda auditiva de grau moderado 6 .

Outro estudo com 2.751 idosos residentes a oeste de Sydney na Australia com o objetivo de avaliar a prevalência de tontura/vertigem mostrou que a prevalência de tontura/vertigem foi de $36,2 \%$. $\mathrm{O}$ zumbido foi associado com tontura e os resultados destacaram que a tontura/vertigem tinha maior impacto sobre a qualidade de vida deles ${ }^{7}$.

Com o decorrer da idade as alterações vestibulares e outros sintomas associados aumentam. Estudo realizado sobre as características clínicas, epidemiológicas e tratamentos realizados com sujeitos idosos com labirintopatias, realizado através do levantamento de 200 prontuários no período de 2008 a 2011, observaram que a idade média dos sujeitos foi de 70,5 anos. As queixas mais apresentadas foram: tontura não rotatória em $74,5 \%$; zumbido $56,5 \%$; perda auditiva $49 \%$; vertigem $9,5 \%(n=19)$; desequilíbrio $5 \%(n=10)$; associação entre tontura e zumbido $2,5 \%(\mathrm{n}=5)$; associação entre labirintopatia e perda auditiva bilateral $30 \%$ $(n=60)$; perda auditiva na orelha direita $6 \%(n=12)$ e perda auditiva na orelha esquerda $13,5 \%(\mathrm{n}=27)$; associação entre labirintopatia e zumbido $56,5 \%$ $(n=113)$. Neste estudo foi observado o aumento relacionado à perda auditiva e zumbido em idosos $\mathrm{Os}$ autores concluíram que os dados epidemiológicos, os sintomas e as comorbidades são importantes para o planejamento clínico e terapêutico desta população idosa para minimizar os impactos decorrentes destas alterações ${ }^{8}$.

A vertigem e a tontura são muito comuns na população idosa. Estudo realizado com 120 idosos acima de 65 anos ou mais com disfunção vestibular, observaram que a tontura rotatória e não rotatória estava presente em $68,3 \%$, sendo que $45,8 \%$ apresentavam a tontura diariamente. Quanto aos sintomas auditivos associados, 70,8\% tinham zumbido, 
$60 \%$ déficit auditivo e $54,2 \%$ sensação de pressão/ plenitude aural. Quanto à queda, foi relatada pelo menos uma queda no último ano em $53,3 \%$ da amostra com disfunção vestibular crônica ${ }^{9}$.

Diante desse cenário, é fundamental o levantamento dos dados do prontuário, para um adequado planejamento clínico e terapêutico desta população idosa com alterações vestibulares e auditivas, visando minimizar os impactos decorrentes e melhora na qualidade de vida.

O objetivo deste estudo é verificar a relação da perda auditiva e queixas relacionadas à tontura, zumbido e dificuldades de comunicação em população idosa.

\section{Material e método}

Esta pesquisa faz parte do projeto intitulado "Relações entre equilíbrio, audição e cognição do idoso", aprovado pelo número 445052/20147 e pelo Comitê de Ética da Universidade sob número CAEE: 43831015.1.0000.5482. Trata-se de estudo retrospectivo descritivo e analítico, cuja coleta de dados foi realizada por meio de consulta a prontuários arquivados no Serviço de Reabilitação Auditiva do município de Jacareí - SP que contava, segundo a Fundação SEADE (2011), com aproximadamente 213.155 habitantes, entre os quais em torno de 23.486 pessoas tinham 60 anos ou mais ( $11,01 \%$ da população total).

Foram identificados e selecionados 468 prontuários de pacientes com idade superior a 60 anos submetidos à avaliação auditiva, cadastrados no setor acima referido, no período de 2011 a 2015.

Foram excluídos prontuários de idosos que apresentaram resultados alterados na imitânciometria com curvas tipos B, C., Ad, As. Foram incluídos na pesquisa todos os idosos com indicação do médico otorrinolaringologista, cadastrados com perda auditiva relacionada à idade, com limiares nas frequências de 250 a $8000 \mathrm{kHz}$ superior a 25 dBNA, aguardando processo de seleção e adaptação de próteses auditivas.

Dos prontuários foram considerados os seguintes dados sócio demográficos: sexo, idade superior a 60 anos e resultados dos exames audiológicos, a saber: grau de perda auditiva e simetria entre as orelhas e se era unilateral ou bilateral. A presença de queixas auto relatadas relacionadas a zumbido, tontura, e ouvir e não entender. Os dados foram analisados isoladamente e não foram associados.

Os dados foram tabulados e processados pelo software SPSS 21.00 Foi aplicado o teste de correlação bivariada com coeficiente de Spearman para análise de relação entre variáveis perdas auditivas, tontura, zumbido e dificuldades de comunicação. Os valores de alfa foram considerados significativos quando menores ou iguais a 0,05 . O valor beta admitido foi de 0,1 .

\section{Resultados}

A amostra foi composta por 295 (63\%) prontuários de sujeitos do sexo feminino e 173 (37\%) do masculino com idade média de 72,7 anos (mínima de 60 anos e máxima de 94 anos e DP 8,64).

O estudo demonstra que no grupo analisado $53,8 \%$ das perdas são classificadas como moderada. A queixa "ouvir e não compreender" foi encontrada em $35,3 \%$ dos idosos seguida por queixa de zumbido $(45,1 \%)$ e tontura $(20,3 \%)$. Observou-se que a tontura tem relação significativa com queixar-se de não entender a fala $(p<0,001 ; R=0,17)$ e entre o zumbido e tontura ( $\mathrm{p}<0,001 ; \mathrm{R}=0,32$ ). Pode-se observar que 77,7\% (364) dos sujeitos apresentaram perdas classificadas como moderada a moderadamente severa, do tipo simétrica (377-80,6\%) e bilateral (453-96,8\%). Quanto às queixas, 45,1\% (211) da amostra referem ter zumbido, 20,3\% (95) tontura e 35,3\% (165) ouvem e não entendem. Conforme demostra tabela 1

Como pode ser visualizado na tabela 2 , ao aplicar o teste de correlação bivariada, verificou-se relação positiva significante entre tontura e as variáveis queixa auto-declarada de dificuldade de entender a fala, presença de zumbido e perdas auditivas assimétricas e bilaterais; e entre dificuldade de entender a fala e as variáveis perdas auditivas assimétricas e bilaterais e zumbido. Verificou-se ainda correlação entre perdas auditivas de grau moderado e moderadamente severo e dificuldade de entender a fala $(\mathrm{P}<0,001 ; \mathrm{R}=0,30)$. 
Tabela 1. Caracterização da amostra idosa segundo o grau da perda auditiva e queixas relacionadas a zumbido, tontura e "ouvir e não entender" ( $n=468)$.

\begin{tabular}{lccc}
\hline Variável & & $\mathbf{n}$ & \% \\
\hline & Caracterização da perda auditiva & 8,1 \\
& Leve & 38 & 53,8 \\
& Moderada & 252 & 23,9 \\
Grau da perda & Mederada severa & 112 & 11,1 \\
& Severa & 52 & 3,0 \\
& Profunda & 14 & 80,6 \\
& Simétrica & 377 & 19,4 \\
& Assimétrica & 91 & 96,8 \\
Zumbido & Bilateral & 453 & 3,2 \\
\hline \multirow{2}{*}{ Tontura } & Unilateral & 15 & 45,1 \\
& & & 54,9 \\
Ouve mas não entende & Presença & 211 & 20,3 \\
& Ausência & 257 & 79,7 \\
& Presença & 95 & 35,3 \\
& Ausência & 373 & 64,7 \\
\hline
\end{tabular}

Tabela 2. $P$ Valores e Coeficiente de correlação $(R)$ da análise entre as variáveis - características da perda auditiva simétrica, perda auditiva bilateral, dificuldade de entender a fala e zumbido.

\begin{tabular}{cccccc}
\hline Variáveis & & Tontura & P.A.B & DEF & Zumbido \\
\hline \multirow{2}{*}{ P.A.B. } & $\mathrm{P}$ & $0,047^{*}$ & - & - & - \\
& $\mathrm{R}$ & 0,092 & - & - & - \\
DEF & $\mathrm{P}$ & $<0,001^{*}$ & $0,042^{*}$ & - & - \\
& $\mathrm{R}$ & 0,172 & 0,094 & - & - \\
Zumbido & $\mathrm{P}$ & $<0,001^{*}$ & 0,515 & $<0,001^{*}$ & - \\
& $\mathrm{R}$ & 0,322 & $-0,03$ & 0,228 & - \\
P.A.S & $\mathrm{P}$ & $0,013^{*}$ & $<0,001^{*}$ & $0,026^{*}$ & 0,244 \\
& $\mathrm{R}$ & 0,114 & 0,217 & 0,103 & $-0,054$ \\
\hline
\end{tabular}

$p=$ Valor de significância; $R=$ Coeficiente de correlação; P.A.B. =Perda auditiva bilateral; P.A.S = Perda auditiva simétrica; D.E.F = Dificuldade Entender Fala *Teste de correlação bivariada (Coeficiente de Spearman)

\section{Discussão}

O fato de ser levantamento de prontuário retrospectivo com queixas audiológicas e otoneurológicas limitou a nossa pesquisa. Apesar de existir atendimento fonoaudiológico e otorrinolaringológico aos idosos, no Serviço de Reabilitação Auditiva, não existe no município uma política de atenção à saúde auditiva do idoso, nem ações ou programas integrais e integrados de atenção à saúde do idoso.

Observamos neste estudo que $53,8 \%$ da amostra apresenta deficiência auditiva moderada e a idade média encontrada foi de 72,73 anos. Este achado corrobora com a pesquisa realizada em São Paulo, com 191 idosos, que encontrou a prevalência de $55 \%$ da população com deficiência moderada e $60 \%$ da amostra tinha a idade média de 75,2 anos ${ }^{6}$.

Nossos achados demonstram que $77,7 \%$ das perdas são classificadas como moderada a moderadamente severa, quadro esse que pode levar a dificuldades de comunicação. É interessante observar que apesar de termos uma porcentagem expressiva de sujeitos com perda auditiva, não encontramos a mesma proporção ao analisarmos a queixa "ouvir, mas não entender" $(35,3 \%)$. Verificou-se, ainda, correlação entre perdas auditivas de grau moderado e moderadamente severo e dificuldade de entender a fala $(\mathrm{P}<0,001 ; \mathrm{R}=0,30)$, o que é esperado, devido ao grau de perda auditiva desta população ${ }^{7,14}$. 
Estudos demostraram que quanto maior a magnitude da perda auditiva, maior a probabilidade de isolamento social. A perda auditiva dessa população tem impacto na comunicação e na qualidade de vida, e o aparelho de amplificação sonora individual, assim como o uso de tecnologia assistiva, pode melhorar a qualidade de vida dessa população, pois traz de volta a sua capacidade de se comunicar e de se inserir de novo na vida social do seu grupo ${ }^{7,10,11}$.

Caso essa situação da perda auditiva não seja revista, enfrentaremos aquilo que Debet denomina de "reprivatização da velhice", ou seja, o afastamento gradual do Estado de sua função de proteção, delegando às famílias, a responsabilidade pelo atendimento às situações de vulnerabilidade física da pessoa idosa ${ }^{12}$.

Neste estudo, verificou-se, ainda, que quase metade da amostra $(45,1 \%)$ apresentou queixa de zumbido e esses achados podem ser comparados (vão na mesma direção) ao estudo realizado no Brasil com 498 sujeitos acima de 60 anos, na qual a prevalência de zumbido foi de $47 \%$ e de outro estudo com 200 idosos, em que a prevalência de zumbido foi de $56,5 \%{ }^{8,20}$.

A queixa "ouvir e não compreender" foi encontrada em $35,3 \%$ de idosos com perda auditiva. Dependendo da circunstância, a compreensão de fala pode exigir mais ou menos esforço auditivo e o reconhecimento da fala piora se houver a presença de ruído de fundo ${ }^{6,14}$.

Tal dado alerta para a necessidade dos serviços fonoaudiológicos focarem sua atuação não apenas nas ações de diagnóstico e reabilitação auditiva. Devem sim ser responsáveis pelo acolhimento dos idosos, acompanhando as dificuldades não só de comunicação, e buscando melhor ajustamento psicossocial dos mesmos ${ }^{4,7,10}$.

Cabe lembrar que esse olhar mais amplo vai ao encontro do que é preconizado pela Classificação Internacional de Funcionalidade, Incapacidade e Saúde (CIF), em que se evidencia o quanto as alterações sensoriais impactam e limitam a vida cotidiana dos sujeitos.

\section{Conclusão}

Com o decorrer da idade, os problemas otoneurológicos aumentam, como podemos observar no nosso estudo, como a queixa ouvir e não compreender observada em $35,3 \%$ dos idosos, a prevalência de zumbido de $45,1 \%$ e $20,3 \%$ de tontura. A partir destes achados, gestores locais poderão elaborar um planejamento adequado, visando à melhoria na qualidade do serviço e de vida dessa população idosa.

\section{Referências}

1. IBGE (Instituto Brasileiro de Geografia e Estatística) http:// www.sdh.gov.br/assuntos/pessoa-idosa/dados-estatisticos/ DadossobreoenvelhecimentonoBrasil.pdf acesso em 3/4/2016).

2. National Institute on Deafness and Other Communication Disorders http://www.nidcd.nih.gov/staticresources/health/ healthyhearing/tools/pdf/HearingLoss.pdf acesso em 3/9/2015.

3. Mick P, Kawachi I, Lin FR. The Association between Hearing Loss and Social Isolation in Older Adults. Otolaryngol Head Neck Surg. 2014; 150(3): 378-84.

4. Dawes P, Emsley R, Cruickshanks KJ, Moore DR, Fortnum $\mathrm{H}$, Edmondson-Jones M, McCormack A, Munro KJ. Hearing loss and cognition: the role of hearing aids, social isolation and depression. PLoS One. 2015; 11: 10-3.

5. Beck DL, Clark JL. Audition matters more as cognition declines: cognition matters more as audition declines. Audiology Today. 2009; 21(2): 48-52.

6. Araujo TM, Iório MCM. Perfil populacional de idosos encaminhados à seleção de próteses auditivas em hospital público. Audiol Commun Res. 2014; 19(1): 45-51.

7. Gopinath B, McMahon CM, Rochtchina E, Mitchell P. Dizziness and vertigo in an older population: the Blue Mountains prospective cross-sectional study. Clinical Otolaryngology. 2009; 34(6): 552-6.

8. Kasse CA, Onichi ET. Caraterísticas clínica de 200 idosos da comunidade com queixas vestibulares. Rev. Bras. Med. 2014; 71 (5): 129-34.

9. Gazzola JM, Ganança FF, Aratani MC, Perracini MR, Ganança MM. Caracterização clínica de idosos com disfunção vestibular crônica. Rev Bras Otorrinolaringol 2006; 72(4): $515-22$.

10. Edwards B. Cognitive Load and Amplification: Our speech- in- noise testing may be telling only part of the story; cognitive load appears to play a major role in patients' success with hearing aids. Research: Hearing \& Cognition. 2013; 10(1).

11. Teixeira AR, Almeida LG, Jotz GP, De Barba MC. Qualidade de vida de adultos e idosos pós-adaptação de próteses auditivas. Rev Soc Bras Fonoaudiol. 2008; 13(4): 357-61.

12. Debert GG. A reinvenção da velhice: socialização e processo de reprivatização da velhice. São Paulo. EDUSP; FAPESP.1999.

13. Anstey KJ, Luszcz MA, Sanchez L. A reevaluation of the common factor theory of shared variance among age, sensory function, and cognitive function in older adults. J Gerontol B Psychol Sci Soc Sci. 2001; 56(1): 3-1

14. Pichora-Fuller M K, Gurjit S. Effects of age on auditory and cognitive processing: implications for hearing aid fitting and audiologic rehabilitation. Trends in amplification. 2006; 10: 29-59.

15. Cacciatore F, Napoli C, Abete P, Marciano E, Triassi M. Quality of life determinants and hearing function in an elderly population: Observatory Geriatric Campano Study Group. Gerontology. 1999; 45(6): 323-8. 
16. Cruickshanks KJ, Klein R, Klein BE, Wiley TL, Nondahl DM. Cigarette smoking and hearing loss: the epidemiology of hearing loss study. JAMA. 1998; 279(21): 1715-9.

17. Lin FR. Hearing Loss and Cognition Among Older Adults in the United States. J Gerontol. 2011; 66(10): 1131-6.

18. Lindenberger U, Baltes PB. Sensory functioning and intelligence in old age: a strong connection. Psychol Aging. 1994; 9(3): 339-55.
19. Southall K, Gagné JP, Jenninngs MB. Stigma: a negative and a positive influence on help-seeking for adults with acquired hearing loss. Ècole dÒrthophonie et d’Audiologie, Université de Montreal, Montréal,Québec,Canada. 2010; 49 (11): 804-14.

20. Gibrin PCD, Melo JJ, Marchiori LLM. Prevalência de queixa de zumbido e prováveis associações com perda auditiva, diabetes mellitus e hipertensão arterial em pessoas idosas. CoDAS 2013; 25(2): 176-80. 